\title{
EFEITO DA IDADE RELATIVA EM NADADORES PARTICIPANTES DO MUNDIAL DE ESPORTES AQUÁTICOS BARCELONA 2013
}

Otávio Gomide Costa, Universidade Federal de Ouro Preto - UFOP, Ouro Preto, Minas Gerais - Brasil

Emerson Filipino Coelho, Universidade Federal de Ouro Preto - UFOP, Ouro Preto, Minas Gerais - Brasil

Francisco Zakaron Werneck, Universidade Federal de Ouro Preto - UFOP, Ouro Preto, Minas Gerais - Brasil

Leandro Vinhas de Paula, Universidade Federal de Ouro Preto - UFOP, Ouro Preto, Minas Gerais - Brasil

Renato Melo Ferreira, Universidade Federal de Ouro Preto - UFOP, Ouro Preto, Minas Gerais - Brasil

\section{RESUMO}

O efeito da idade relativa é definido como a vantagem que atletas nascidos no início do ano de seleção podem apresentar sobre os atletas nascidos no final do mesmo ano. Atletas nascidos no início do ano possuem maior idade cronológica e probabilidade de estarem em estágios de maturação biológica mais avançados. O objetivo foi analisar o efeito da idade relativa em nadadores participantes do Mundial de Esportes Aquáticos de Barcelona 2013. Foram analisados os quartis de nascimento, por meio da coleta das datas de nascimento dos atletas no site da FINA. Os resultados apontam que na amostra, como um todo, o EIR não foi observado, mas quando analisado a especificidade da prova observou-se maior concentração de atletas nascidos nos três primeiros quartis. Conclui-se que o EIR foi identificado em relação à especificidade das provas. Novos estudos são necessários em outros níveis e competições de natação para melhor caracterização do EIR na natação.

Palavras-Chave: Efeito da idade relativa; Atletas; Natação.

\section{RELATIVE AGE EFFECT ON PARTICIPANTS IN THE WORLD SWIMMERS OF WATER SPORTS BARCELONA 2013}

\begin{abstract}
The relative age effect is defined as the advantage that athletes born in the beginning year selection may have on athletes born later that same year. Athletes born earlier have higher chronological age and likely to be in more advanced biological maturation stages. The aim was to analyze the relative age effect on swimmers in the World Championship Barcelona 2013. Quartiles of birth were analyzed by collecting the dates of birth of the athletes on the FINA website. The results show that the sample, the RAE was not observed, but when analyzing the specificity there was a higher concentration of athletes born in the first three quartiles. It is concluded that the RAE was identified in relation to
\end{abstract}


the specificity of swimmers. Further studies are needed at other levels and swimming competitions to better characterize the RAE in swimming.

Key-Words: Relative age effect; Athletes; Swimming.

\section{EFECTO DE LA EDAD RELATIVA DE LOS PARTICIPANTES EN LOS NADADORES DEL MUNDO DE DEPORTES DE AGUA BARCELONA 2013}

\section{RESUMEN}

El efecto de la edad relativa (Relative Age Effect - RAE) se define como la ventaja de que los atletas nacidos en el comienzo de la selección pueden tener en los atletas nacidos ese mismo año. Los atletas nacidos a principios de este año tienen mayor edad cronológica y la probabilidad de estar en las etapas de maduración biológicos más avanzados. El objetivo fue analizar el efecto de la edad sobre nadadores participantes en Mundial de Natación en Barcelona 2013. Cuartiles de nacimiento fueron analizados mediante la recopilación de las fechas de nacimiento de los atletas en la página web de la FINA. Los resultados muestran que la muestra en su conjunto, el RAE no se observó, pero cuando el análisis de la especificidad de la prueba hubo una mayor concentración de los atletas nacidos en los tres primeros cuartiles. Se concluye que el RAE se identificó en relación con la especificidad de las pruebas. Se necesitan más estudios en otros niveles y competencias de natación para caracterizar mejor el RAE en la natación.

Palabras Clave: Efecto de la edad relativa; Atletas; Natación. 


\section{INTRODUÇÃO}

A natação desportiva é definida como o deslocamento corporal mais rápido possível na água, produto da interação entre as forças de arrasto (drag) promovidas pela ação dinâmica do fluido e de sustentação (lift) perpendicular ao fluxo de fluido. ${ }^{1}$ A natação foi inserida nos primeiros Jogos Olímpicos da Era Moderna em 1896 e as provas disputadas foram os 100, 500 e 1200 metros livre. ${ }^{2}$ Durante as décadas seguintes, principalmente após a fundação da Federação Internacional de Natação - FINA em 1908, outras provas foram incluídas junto ao processo de composição desta modalidade, tais como 50, 200, 400, 800 e 1500 metros livre, havendo também o processo de divisão de categorias etárias. ${ }^{3}$

A divisão das categorias esportivas da natação é feita a partir do ano de nascimento do atleta. Para a SWAG - Age Group Rules (Regras de Faixa Etárias), as regras de idade e os critérios de divisão definidos pelas confederações, ${ }^{3}$ dessa forma, no Brasil as categorias competitivas são divididas em Mirim 1 (9 anos), Mirim 2 (10 anos), Petiz 1 (11 anos), Petiz 2 (12 anos), Infantil 1 (13 anos), Infantil 2 (14 anos), Juvenil 1 (15 anos), Juvenil 2 (16 anos), Junior 1 (17 anos), Junior 2 (18 e 19 anos), Sênior (20 anos ou mais). ${ }^{4}$ Tais divisões supracitadas, são disputadas em competições de forma separada ou em conjunto. Já em um campeonato mundial ou Jogos Olímpicos, por exemplo, várias categorias etárias podem estar inseridas em uma mesma competição.

Dentro do contexto histórico de algumas modalidades esportivas, muitos atletas se destacam, em função do seu desempenho esportivo em determinadas categorias. Pesquisas mostram que a idade interfere no resultado da competição sendo que alguns estudos apontam que a superioridade pode estar relacionada a partir de alguns fatores, entre eles, o mês de nascimento. ${ }^{5-6}$ A diferença na idade cronológica entre atletas da mesma categoria etária é chamada de idade relativa. A idade relativa pode favorecer o desempenho em determinadas categorias esportivas, já que algumas capacidades físicas e antropométricas podem ser desenvolvidas mais cedo por atletas que nascem no início do ano (meses de janeiro a março) quando comparados com atletas que nascem nos meses finais do mesmo ano (outubro a dezembro). ${ }^{7}$ Sendo assim, atletas nascidos no início do ano esportivo, podem se beneficiar nos processos de seleção esportiva. Essa possível vantagem é intitulada como efeito da idade relativa (EIR). 
O EIR é definido como a possível vantagem que os atletas nascidos mais próximos ao início do ano de seleção levam em relação a seus pares nascidos posteriormente. ${ }^{8}$ A causa mais frequente deste efeito é a maturação biológica, uma vez que os atletas relativamente mais velhos na maioria das vezes se encontram em estágios maturacionais mais avançados quando comparados aos atletas relativamente mais novos.

A maturação biológica ocorre durante o desenvolvimento etário do indivíduo, podendo ser precoce ou tardio quando relacionado com o esporte, pois, a mesma pode condicionar o processo de seleção e exclusão de atletas e não atletas no âmbito desportivo. ${ }^{9}$ Tais processos começam próximo aos 5 anos de idade em algumas modalidades esportivas, como por exemplo a ginástica. ${ }^{6}$ É importante ressaltar também que, no sexo masculino, a maturação tardia beneficia o desempenho e o sucesso em algumas modalidades, como no basquete e atletismo, onde o desenvolvimento esquelético propício para estes esportes ocorre entre 16 e 18 anos, enquanto o sexo feminino, a maturação está diretamente relacionada a menarca podendo ocorrer mais precocemente. ${ }^{10}$

Outro ponto importante que deve ser levado em consideração para a análise do efeito da idade relativa é a caracterização da modalidade esportiva. Carli et al. ${ }^{11}$ analisaram o EIR em atletas de futebol das categorias sub 17 e sub 20 da Europa e América do Sul. Os resultados apontam que este fenômeno está presente no grupo avaliado. Os autores ainda destacam que por ser uma modalidade caracterizada, principalmente, pela agilidade, velocidade, domínio de espaço temporal e contatos físicos constantes, favorece o processo de seleção de atletas mais fortes e desenvolvidos fisicamente. Além disso, Penna et al. ${ }^{12}$ apontam que tais desigualdades entre estes grupos de atletas (mais e menos desenvolvidos) da mesma idade, favorecem os atletas nascidos no primeiro semestre do processo de seleção nas categorias iniciantes de futebol, o que caracteriza o efeito da idade relativa.

Em contrapartida, em um estudo realizado com atletas de Taekwondo participantes dos Jogos Olímpicos, Albuquerque et al. ${ }^{13}$ apontam que não foi identificado o EIR em relação a esta modalidade. Uma das justificativas apontadas no estudo foi que o Taekwondo é relativamente um esporte novo no contexto olímpico, e dessa forma, o número de praticantes nas categorias iniciantes é menor, reduzindo assim a relevância do fator 
diferença de idade. Ao comparar o número de praticantes da modalidade, não chega ser expressivo, quando comparado ao de outras modalidades, como o futebol. Este fato pode gerar a não diferenciação do grupo durante o processo de seleção e consequentemente a não identificação do EIR. Quanto maior o número de praticantes de uma mesma categoria em uma dada modalidade, maior a possibilidade de ocorrer o EIR, já que a concorrência para obter um espaço na equipe ou seleções, será maior. ${ }^{6}$

Ao analisar os trabalhos realizados sobre o EIR no esporte, constata-se que a maioria destes, foi desenvolvida com modalidades coletivas. ${ }^{14,15}$ No entanto, existe uma necessidade de avaliar tal efeito em modalidades individuais, como a natação. ${ }^{16}$ Deste modo questiona-se se o EIR está presente em nadadores participantes do Campeonato Mundial de Esportes Aquáticos Barcelona 2013.

A especificidade na natação é determinada por algumas capacidades, como a força e potência para os velocistas e resistência aeróbica para os fundistas. ${ }^{1}$ Um dos poucos trabalhos identificados com a natação competitiva foi o de Ryan ${ }^{16}$ que investigou o EIR na natação, futebol, voleibol e handebol. O EIR se fez presente em todas as modalidades, porém na natação, apenas no sexo feminino as diferenças foram significativas. Segundo o autor, a amostra de competidores do sexo masculino era menor, fato que influenciou diretamente o resultado.

A relevância do presente estudo está na preocupação de estabelecer se o efeito da idade relativa está presente quando se analisa a especificidade competitiva dos atletas de natação e a excelência esportiva, uma vez que os atletas olímpicos são produtos de processos de seleção realizados por parte de treinadores. Torna-se importante verificar se a idade relativa é um dos fatores determinantes para chegar ao alto nível.

Portanto o objetivo do estudo foi verificar a existência do efeito da idade relativa em nadadores participantes do Campeonato Mundial de esportes aquáticos de Barcelona em 2013, analisando possíveis diferenças entre os sexos, idade, continente, especificidade de provas e excelência esportiva. Assume-se como hipótese que o EIR está presente nestes 
atletas, especialmente no sexo feminino, e que há diferenças dependendo da prova/categoria e continente (país) ao qual o atleta pertence.

\section{MÉTODOS}

Amostra

A amostra foi composta por nadadores que conquistaram classificação para o Campeonato Mundial de Esportes Aquáticos Barcelona 2013 através de seus índices, sendo que, o critério de inclusão foi que os competidores deveriam figurar entre os 30 primeiros colocados de cada prova, totalizando 519 atletas do sexo masculino e 509 atletas do sexo feminino, com idade de 22,9 $\pm 3,4$ anos. O presente trabalho seguiu os mesmos procedimentos metodológicos adotados em trabalhos nacionais ${ }^{11}$ e internacionais. ${ }^{13}$

\section{Procedimento}

Este trabalho consistiu numa pesquisa quantitativa e descritiva. Primeiramente, as datas de nascimento foram obtidas no site oficial da Federação Internacional de Natação (www.fina.org), ${ }^{3}$ onde está disponível juntamente com os balizamentos e resultados da natação do Mundial de Esportes Aquáticos Barcelona 2013. Como critério de seleção, foram selecionados para análise os 30 primeiros colocados em cada prova. Tal critério se justifica pela tentativa de se avaliar apenas os atletas que possuíssem índice de participação de campeonato (Índice A), diminuindo a chance de atletas com índices B ou convidados constarem na amostragem do estudo, o que, dessa forma, não representaria a avaliação do EIR na excelência pretendida.

Os dados foram extraídos e arquivados em uma tabela de Excel. As datas de nascimento bem como outras variáveis foram evidenciadas para supostas comparações, tais como, sexo, idade, especificidade da prova e desempenho esportivo. O desempenho dos atletas se avaliou a partir do número de medalhas conquistadas na competição e suas posições na classificação geral. A data de nascimento destes atletas foi agrupada em quartis divididos em $1^{\circ}$ quartil (Janeiro, Fevereiro e Março), $2^{\circ}$ quartil (Abril, Maio e Junho), $3^{\circ}$ quartil (Julho, Agosto e Setembro) e $4^{\circ}$ quartil (Outubro, Novembro e Dezembro). 
Análise Estatística

Para testar o efeito da idade relativa, foi realizado o teste de qui-quadrado $\left(\chi^{2}\right)$, para a comparação da distribuição esperada e observada nos quartis de nascimento dos atletas. Conforme estudos anteriores, os valores esperados foram calculados assumindo igual distribuição de nascimentos em cada quartil do ano. Uma posterior análise de proporção 2x2 com correção de Bonferroni entre cada quartil foi realizada para encontrar onde estavam as possíveis diferenças. Todos os testes foram feitos utilizando-se o programa SPSS 19.0 para Windows, utilizando nível de significância de 5\%.

\section{RESULTADOS}

Os resultados encontrados referentes à distribuição dos quartis de nascimento de todos os atletas, por sexo, tipo de prova, etapa e medalhistas encontram-se na Tabela 1. Foi observado maior percentual de atletas nascidos no $1^{\circ}$ e $3^{\circ}$ quartis, comparados ao $2^{\circ}$ e $4^{\circ}$ quartis, embora a diferença tenha ficado próxima ao nível de significância $(\mathrm{p}=0,08)$. $\mathrm{O}$ EIR não foi verificado nos atletas do sexo masculino, e ficou marginalmente significativo nas atletas do sexo feminino $(\mathrm{p}=0,08)$.

Tabela 1 - Avaliação dos quartis de nascimento dos atletas do Mundial de Esportes Aquáticos de Barcelona 2013 por sexo, tipo de prova (especificidade), etapa, expertise (medalhista), através do teste qui-quadrado.

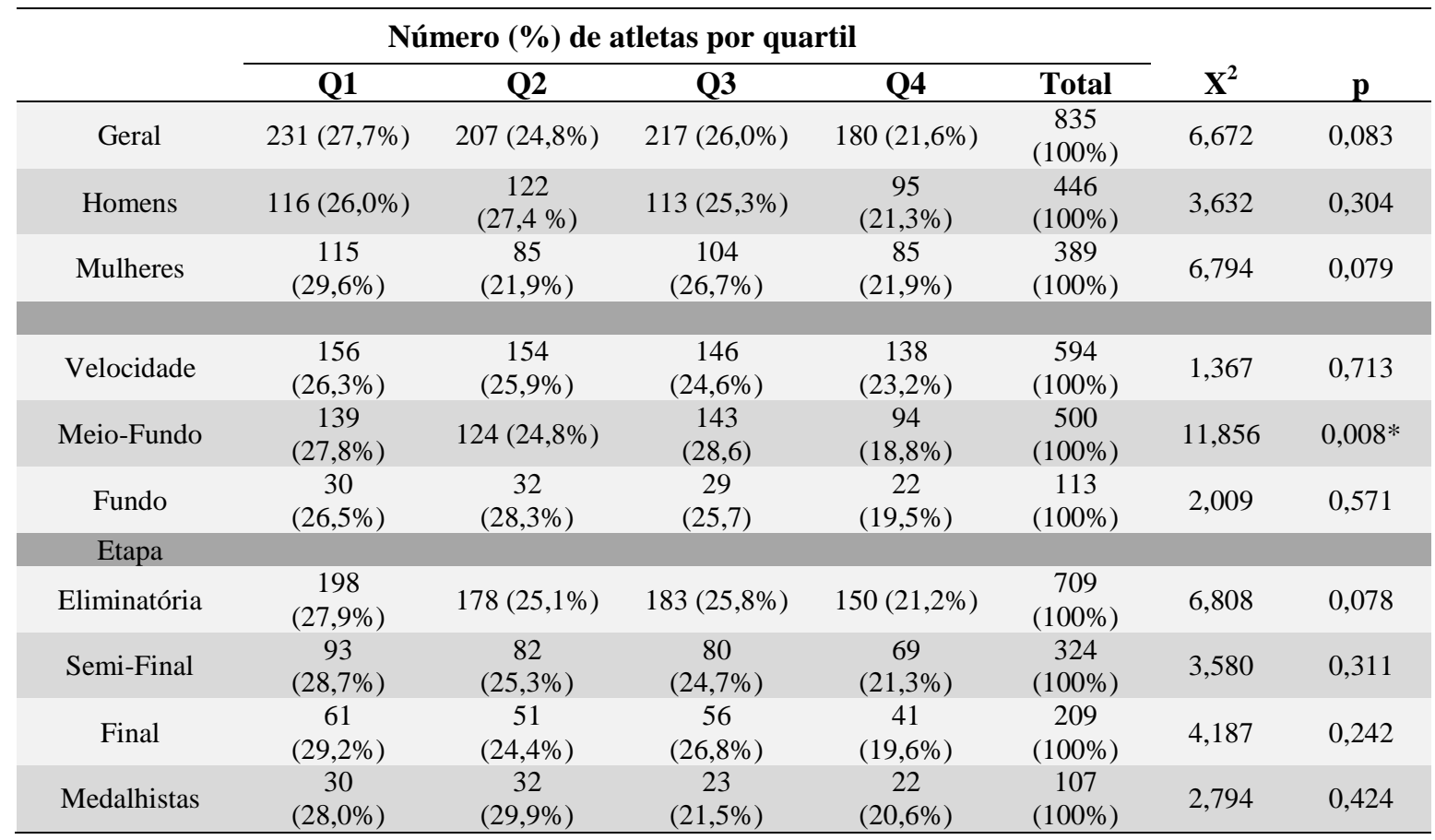

Conexões: revista da Faculdade de Educação Física da UNICAMP, Campinas, v. 13, n. 2, p. 83-97, abr./jun. 2015. ISSN: 1983-9030 
Quanto ao tipo de prova, observou-se menor percentual de atletas nascidos no $4^{\circ}$ quartil nas provas de meio-fundo $(\mathrm{p}=0,008)$ - Figura 1. Nas provas de velocidade e fundo, não foi observado o EIR ( $>0,05)$. Não houve diferença significativa entre os quartis de nascimento dos atletas semifinalistas e finalistas. Quando se analisou as etapas, nas eliminatórias as diferenças também se aproximaram do nível de significância $(p=0,078)$. Ao analisar o EIR por continente, não foi identificada diferença significativa para os 7 países mais representativos (histórico de medalhas em campeonatos mundiais) participantes do Mundial.

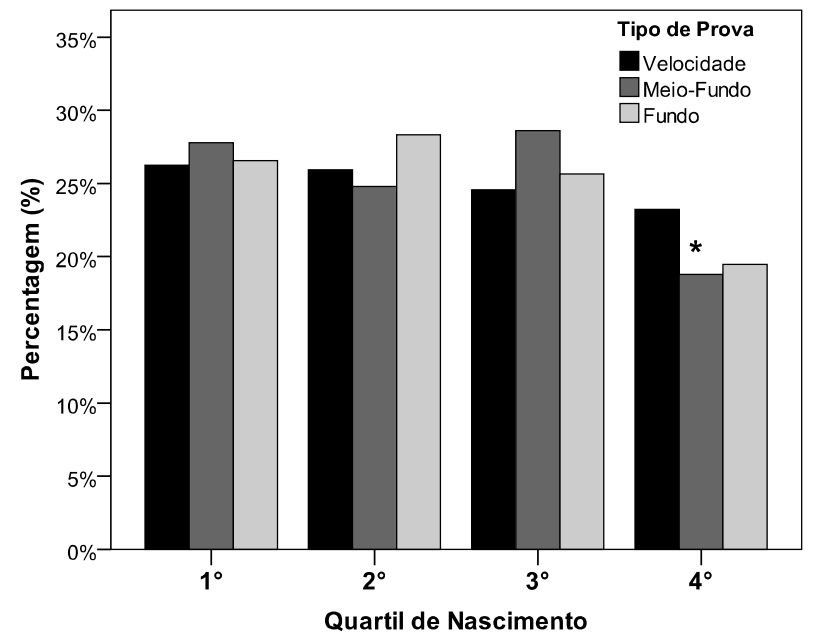

Figura 1: Distribuição percentual dos quartis de nascimento dos atletas de natação que disputaram o Campeonato Mundial de Natação de Barcelona $2013(\mathrm{n}=107)$ por tipo de prova. (*diferença significativa $1^{\circ}, 2^{\circ}$ e $3^{\circ}$ quartis vs. $4^{\circ}$ quartil, $\mathrm{p}=0,008$ ).

Porém, a análise da distribuição do semestre de nascimento dos atletas medalhistas $\left(1^{\circ}, 2^{\circ}\right.$ e $3^{\circ}$ lugares) revelou uma maior proporção de atletas nascidos no $1^{\circ}$ semestre comparado ao $2^{\circ}$ semestre, embora a diferença não tenha sido estatisticamente significativa - Figura 2. 


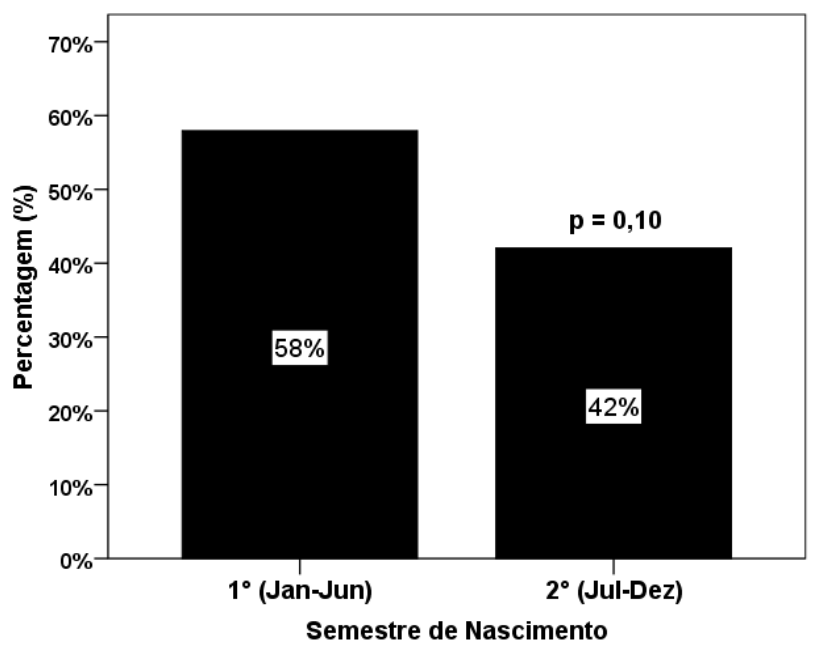

Figura 2: Distribuição percentual do semestre de nascimento dos atletas de natação medalhistas $\left(1^{\circ}, 2^{\circ}\right.$ e $3^{\circ}$ lugares $)$ que disputaram o Campeonato Mundial de Natação de Barcelona 2013 ( $\mathrm{n}=107)$. (Sem diferença significativa entre os semestres, $\mathrm{p}=0,10$ ).

\section{DISCUSSÃO}

Este estudo teve como objetivo verificar o efeito da idade relativa em nadadores participantes do Mundial de Esportes Aquáticos Barcelona 2013, no qual, o tamanho da amostra pode ter influenciado nos resultados. Alguns estudos sugerem que a quantificação da amostra, é um dos fatores inerentes para o resultado. Ryan, ${ }^{16}$ por exemplo, analisou o EIR em nadadores de nível escolar do sexo masculino e feminino, não encontrando diferenças significativas em relação ao sexo masculino possivelmente devido a quantificação da amostra deste gênero, em contrapartida, no sexo feminino foi encontrado o EIR o que pode ter sido influenciado pelo tamanho da amostra, o que corrobora, em parte, os resultados deste estudo.

O EIR em nadadores também pode estar relacionar ao estágio maturacional dos atletas, já que aqueles com maturação biológica precoce (antes dos 13 anos) possuem vantagens físicas e antropométricas em comparação aos seus pares de mesma idade cronológica com ritmo maturacional normal ou tardio. ${ }^{17}$ Porém, pressupõe-se que nos nadadores do sexo masculino a competição em si, pode gerar cargas de motivação em relação à busca por conquistas de resultados em todas as circunstâncias independente da data de nascimento, o que diminuiria o EIR, ao analisar o sexo. Nazario et al. ${ }^{18}$ realizaram um estudo no qual Conexões: revista da Faculdade de Educação Física da UNICAMP, Campinas, v. 13, n. 2, p. 83-97, abr./jun. 2015. ISSN: 1983-9030 
identificou que os homens são mais motivados e autodeterminados, superando assim dificuldades impostas pelo ambiente, ao se comparar com o sexo feminino.

Já no sexo feminino, para Malina et al. ${ }^{10}$ a maturação interfere diretamente no desempenho esportivo já que relatam que algumas medidas e variações antropométricas estão interligadas à aptidão física. ${ }^{19}$ No presente estudo, o sexo feminino apresentou uma distribuição maior das atletas no primeiro e terceiro quartis comparados ao segundo e quarto, mesmo o EIR ficando próximo do nível de significância $(\mathrm{p}=0,08)$. Uma das possíveis explicações para este resultado foi à avaliação das 30 primeiras atletas de cada prova somente. Espera-se que ao se avaliar todas as atletas, inclusive aquelas que ficaram a partir da $31^{\circ}$ posição, possa se identificar o EIR neste grupo, uma vez que as diferenças cronológicas etárias poderiam ser maiores devido a uma suposta inferioridade de desempenho relacionado à maturação destas atletas.

Ao analisar o tipo de prova, o EIR foi verificado em provas de meio fundo, enquanto, que nas provas de fundo e velocidade, tal efeito não foi evidenciado. As provas de 200 e 400 metros apresentaram o maior número de atletas que disputaram tais distâncias, ao se comparar com as provas de velocidade $(50 \mathrm{~m}$ e $100 \mathrm{~m})$ e fundo $(800 \mathrm{~m}$ e $1500 \mathrm{~m})$. Dessa forma, pode ter se identificado o EIR nestas provas pelo número de atletas participantes. Outro ponto para este resultado é que os atletas meio fundistas podem exigir maiores capacidades físicas inerentes à modalidade, por isso, torna-se importante durante o processo de seleção, pois os treinadores tendem a selecionar e dar mais atenção para os atletas com melhores condicionamentos físicos.

Já em relação aos finalistas e semifinalistas, não foram encontrados EIR por segmento. Estes segmentos são compostos por eliminatórias, semifinais e finais, realizados a partir de balizamentos. ${ }^{3-4}$ Diante da competitividade de se atingir bons resultados nestes segmentos, as próprias características da competição podem diminuir o EIR, sendo que, todos os nadadores chegam nesta fase nos mesmos níveis de motivação, em busca das melhores posições e vitórias. O nível de experiência está relacionado com a motivação intrínseca do atleta, ${ }^{20}$ em competições de alto nível, como campeonatos mundiais, acredita-se, que a motivação intrínseca dos atletas esteja alta, devido à iniciativa de atingir metas as metas 
estabelecidas. Sales et al. ${ }^{21}$ avaliaram a motivação de atletas da seleção brasileira nos Jogos Mundiais Escolares, no qual, o estudo indica que os fatores motivacionais intrínsecos, como atingir objetivos e experiências estimulantes, foram mais significativos quando comparados à motivação extrínseca e desmotivação.

Em relação aos atletas medalhistas, não foi identificado o EIR, apesar de uma maior representação dos atletas no primeiro semestre. Ferreira et al. ${ }^{22}$ verificou o contexto do desenvolvimento expert dos nadadores medalhistas olímpicos brasileiros. Os principais resultados apontam que a motivação intrínseca foi fundamental para o desenvolvimento do atleta, e a motivação extrínseca, como por exemplo, medalha, família e treinadores, assim como os adversários, auxiliaram no processo de desenvolvimento de cada atleta. É importante destacar que o grupo analisado é heterogêneo, de forma que, existem atletas nascidos em quartis diferentes, o que pode ter contribuído para os resultados. Os autores concluem que a motivação intrínseca e extrínseca, foi determinante para a melhoria da performance dos atletas. Sugere-se também que durante o processo de formação aqueles atletas que são escolhidos pelos técnicos recebem mais feedbacks e treinos de qualidade, com isso estes podem se sentir motivados para prática da modalidade, enquanto os nãos selecionados podem abandonar o esporte.

Os atletas nascidos nos primeiros quartis podem demonstrar maiores capacidades físicas, pois são mais maduros biologicamente, o que os privilegiam na seleção para competições e na obtenção de bons resultados, informação que corrobora alguns estudos. ${ }^{11,23-25}$, No presente estudo, mesmo em algumas variáveis que não tiveram diferenças significativas, percebe-se que o EIR está presente em todas as análises.

Para análise do EIR por continente, foram selecionados 7 países, Estados Unidos, Austrália, Itália, Japão, China, Alemanha e Grã-Bretanha, que desenvolvem um processo de seleção criterioso e que tem representatividade por meio da expertise em resultados nas competições nos últimos anos. Não foram identificadas diferenças significativas quanto ao EIR. Este fato pode ser justificado, pois o processo de seleção estabelece índices que podem ser alcançados por qualquer atleta, já que a competição não há restrição de idade, ou seja, em qualquer idade pode ser alcançado o índice, minimizando o EIR neste aspecto. 
Este estudo apresentou como limitação a amostragem, ao analisar as provas, de acordo com suas especificidades técnicas. Sugere-se a avaliação do EIR em atletas participantes do Campeonato Mundial Júnior de Natação com a finalidade de identificar o EIR neste grupo. Também sugere-se novos estudos comparando o EIR com variáveis morfológicas, aptidão física de jovens atletas.

Apesar dos resultados não serem significativos, em quase todas as variáveis, houve maiores distribuições no primeiro e segundo quartil. Estes atletas nascidos nos primeiros quartis podem ter desenvolvido picos de maturação mais rápidos, levando vantagem no processo de seleção e atingindo altos níveis de excelência.

\section{CONCLUSÃO}

Conclui-se que o EIR foi identificado na natação quando se analisou a especificidade das provas, sendo constatada menor proporção de atletas nascidos no quarto quartil nas provas de meio fundo. Além disso, não foi identificado este fenômeno em relação ao país de origem dos atletas. No entanto, parece ser mais evidente no sexo feminino. Quanto às implicações práticas deste estudo, sugere-se que os treinadores sejam conscientizados do EIR em razão das consequências negativas que este fenômeno pode trazer.

\section{REFERÊNCIAS}

${ }^{1}$ MAGLISCHO, E. W. Nadando o mais rápido possível. 3. ed. Barueri: Manole, 2010.

${ }^{2}$ SAAVEDRA, J. M.; ESCALANTE, Y.; RODRIGUES, F. A. A evolução da natação. Lecturas, Educacion Fisica y Deportes, Buenos Aires, v. 9, n. 66, 2003.

${ }^{3}$ FEDERAÇÃO INTERNACIONAL DE NATAÇÃO (FINA). Histórico das provas oficiais. Disponível em: http://www.fina.org/H2O/. Acesso em: 17 nov. 2013.

\section{${ }^{4}$ CONFEDERAÇÃO BRASILEIRA DE DESPORTOS AQUÁTICOS. Categorias da} natação. Disponível em: http://www.cbda.org.br/cbda/natacao/ regulamentos. Acesso em: 19 nov. 2011. 
${ }^{5}$ MEDIC, N. et al. Gender, age, and sport differences in relative age effects among US masters swimming and track and field athletes. Journal of Sports Sciences, London, v. 27 , n. 14, p. 1535-1544, 2009.

${ }^{6} \mathrm{MUSH}, \mathrm{J}$; GRODIN, S. Unequal competition as an impediment to personal development: a review of the relative e age effect in sport. Development Review, New York, v. 21, n. 2, p. 147-167, 2001.

${ }^{7}$ COSTA, A. M. et al. The relative age effect among elite youth competitive swimmers. European Journal of Sports Science, v. 13, n. 5, p. 437-444, 2012.

${ }^{8}$ GONAUS, C.; MULLER, E. Using physiological data to predict future career progression in 14- to 17-year-old Austrian soccer academic players. Journal Sports Sciences, London, v. 30, n. 15, p. 1673-1682, 2012.

${ }^{9}$ MALINA, R.; BOUCHARD, C.; BAR-OR, O. Crescimento, maturação e atividade física. 2. ed. São Paulo: Phorte, 2009.

${ }^{10}$ MALINA, R. et al. Skeletal maturation, somatic growth and physical fitness in girls 6-16 of age. International Journal of Sports Medicine, Stuttgart, v. 18, n. 6, p. 413-419, 1997.

${ }^{11}$ CARLI, G. C. et al. Efeito da idade relativa no futebol. Revista Brasileira de Ciência e Movimento, Brasilia, v. 3, n. 17, p. 25-31, 2009.

${ }^{12}$ PENNA, E. M. et al. A. Efeito da idade relativa no futsal de base de Minas Gerais. Revista Brasileira Ciência do Esporte, v. 34, n. 1, p. 41-51, 2012.

${ }^{13}$ ALBUQUERQUE, M. R. et al. Relative age effect in olympic taekwondo athletes. Perceptual \& Motor Skills, Missoula, v. 114, n. 2, p. 461-468, 2012. 
${ }^{14}$ DELORME, N.; RASPAUD, M. The relative age effect in young french basketball players: a study on the whole population. Scandinavian Journal of Medicine e Science in Sports, Oxford, v. 19, n. 2, p. 235-242, 2009.

${ }^{15}$ LIDOR, R. et al. Relative age effect and birthplace effect in division 1 female ballgame players: the relevance of sport-specific factors. International Journal of Sport and Exercise Psychology, Morgantown, v. 12, n. 1, p. 19-33, 2012.

${ }^{16}$ RYAN, P. The relative age effect on minor sport participation: faculty of graduate studies and research. 1989. 121 f. Thesis. (Master Degree) - McGill University Montreal, Canadá, 1989.

${ }^{17}$ MALINA, R. et al. Height, mass and skeletal maturity of elite Portugueses soccer players aged 11 - 16 years. Journal of Sports Sciences, London, v. 18, n. 9, p. 685-693, 2000.

${ }^{18}$ NAZARIO, P. F. et al. Níveis de motivação em nadadores: Uma comparação em relação a autoeficácia, sexo e categoria da modalidade. Revista Biomotriz, Cruz Alta, v. 7, n. 1, p. 29-41, 2003.

${ }^{19}$ RÉ, A. H. N. Crescimento, maturação e desenvolvimento na infância e adolescência: Implicações para o esporte. Motricidade, Ribeira de Pena, v. 7, n. 3, p. 55-67, 2011.

${ }^{20}$ FARIA, T. G. Comparative analysis of intrinsic motivation level of athletes between 16 to 18 years of age who are participants of team and individual sports with different levels of experience. FIEP Bulletin, Foz do Iguaçú, v. 78, n. 1, p. 162-164, 2008.

${ }^{21}$ SALES, E. F. et al. Análise da motivação em atletas da seleção brasileira nos jogos mundiais escolares. Coleção Pesquisa em Educação Física, Várzea Paulista, v. 9, n. 2, p. 45-50, 2010.

${ }^{22}$ FERREIRA, R. M. et al. Nadadores medalhistas olímpicos: Contexto do desenvolvimento brasileiro. Motriz, Rio Claro, v. 18, n. 1, p. 130-142, 2012. 
${ }^{23}$ PENNA, E. M.; MORAES, L. C. C. A. Efeito relativo da idade em atletas brasileiros de futsal de alto nível. Motriz, Rio Claro, v. 16, n. 3, p. 658-663, 2010.

${ }^{24}$ BAXTER-JONES, A. et al. Growth and development of male gymnasts, swimmers, soccer and tennis players: a longitudinal study. Annals of Human Biology, London, v. 22, n. 5, p. 381-394, 1995.

${ }^{25}$ BRANSLEY, R. H.; THOMPSON, A. H.; BARNSLEY, P. E. Hockey success and birth date: the relative age effect. International Review for the Sociology of Sport, Warsaw, v. 45 , n. 1, p. 507-512, 2010.

Recebido em: 06 ag. 2014

Aceito em: 30 mar. 2015

Contato: otavio.g.costa@bol.com.br 